\title{
SISTEMA DE SECADO ARTIFICIAL DE MADERA POR DESHUMIDIFICACIÓN UNA OPCIÓN PARA PEQUEÑOS PRODUCTORES DE MADERA DE LA REGIÓN DE AYSÉN
}

\author{
Bustamante, Jorge ${ }^{14}$; Salinas Jaime y Moya, Iván
}

\section{RESUMEN}

El Instituto Forestal (INFOR) sede Patagonia está ejecutando desde octubre del año 2017 el Programa Prototipo de Transferencia Tecnológica Forestal (PTTF), financiado por el Gobierno Regional de Aysén a través del Fondo Nacional de Desarrollo Regional (FNDR). Este programa tiene como objetivo principal aumentar la competitividad de los actores forestales regionales, a través de la transferencia tecnológica forestal innovadora, integral y continua.

El fortalecimiento de las capacidades tecnológicas en las pequeñas y medianas empresas es de gran importancia para mejorar la competitividad y eficiencia en sus procesos productivos, para que puedan generar valor agregado a los productos derivados de la madera, siendo el secado de la madera fundamental en la industria secundaria para la elaboración y remanufactura.

Según el último censo de la industria primaria existe un total de 49 unidades productivas, solo 32 aserraderos se encuentran trabajando en la Región de Aysén, y solo el 9\% tiene una segunda línea de producción con elaboración o remanufactura. Esta realidad, sumada a la falta de disponibilidad de capital de trabajo y de mano de obra capacitada, ha limitado un desarrollo local de productos derivados de madera para la carpintería y mueblería, y también para la construcción en madera.

La implementación de nuevas tecnologías de secado artificial puede constituirse como una estrategia para dinamizar la economía del sector forestal regional. Este tipo de alternativa de generación de valor ha sido un desafío para INFOR que, durante el año 2014, a través de un proyecto FIA, implementó en la propiedad de un productor maderero un secador artificial por cámara (convencional) en forma exitosa, el cual se encuentra funcionando hasta ahora. Sin embargo, no se ha logrado cubrir la demanda actual de madera seca a nivel local, lo que da cuenta de una necesidad a corto plazo que es necesario resolver. Es por esto, que a través del PTTF se logró adquirir un equipamiento para implementar un sistema de secado de madera bajo un proceso de deshumidificación, una tecnología que data de la década de los años 70 , pero que en la ciudad de Coyhaique y en la región actualmente está poco difundido. El sistema de deshumidificación es una opción concreta de secado de madera para pequeñas producciones.

Este documento presenta una alternativa de secado artificial para pequeños productores de madera a través de un sistema eléctrico por deshumidificación en la Región de Aysén.

Palabras clave: Secado, Deshumidificación, Madera Nativa, Región de Aysén.

\footnotetext{
${ }^{14}$ Instituto Forestal, sede Patagonia, Coyhaique, Chile. jbustamante@infor.cl
} 


\section{SUMMARY}

Under the Aysén Regional Government support the Forestry Institute (INFOR) is developing a Technology Transfer Programme to improve the regional sawnwood producers competitiveness. The small and medium enterprises strengthening is an important matter to increase their produtive processes efficiency in order to incorporate added value to their products and the wood drying is a fundamental tool for the secondary industry

According to the last primary industry census, there is a total o 49 productive units in the region, only 32 sawmills are currently active and only $9 \%$ of which have a second production line for wood elaboration. This fact, joined to the economical resources and trained labour lack, is a serious local development limitation to the production of wood derived products to carpentry, furniture and also wood construction.

New artificial wood drying technologies can be a good strategy to drive the regional forestry sector economy and this alternative has been a chalenge for INFOR. In 2014, INFOR has introduced to a producer property a wood dryer with conventional chamber in a successful way, wich is in operation until now.

However, it has been not possible to cover the local dry wood demand and this is an important need to solve in the short term. Though the Tecnology Transfer Programme INFOR bought an aquipment to implement a wood dryier with a dehumidification system wich is a concrete option to dry wood in small productions.

This paper presents an alternative to artificial wood drying for small producers through an electric dehumidification system in the Aysén Region.

Key words: Wood drying, Dehumidification, Native species, Aysén Region. 


\section{INTRODUCCIÓN}

En Chile, la superficie de bosque nativo es de $14.633 .778,8$ ha y la región concentra el $31 \%$ del total de bosque nativo del país.

La superficie de la Región de Aysén es de 10,7 millones de hectáreas, lo que equivale al $14,3 \%$ del territorio nacional y la ubica como la tercera región más grande del país (CONAF, 2012).

De la superficie regional, el $41,28 \%$ (4.431.845 ha) está cubierta por bosques y solo el $0,33 \%$ (35.671 ha) corresponde a plantaciones forestales.

En la región se encuentran cuatro tipos forestales, predominando el Tipo Forestal Siempreverde, que cubre 1.899.864 ha y corresponde al 54,2\% del nivel nacional. El Tipo Forestal Lenga ocupa una superficie de 1.400 .378 ha, seguido del Tipo Forestal Coihue de Magallanes con 939.169,3 ha y, con menor participación, el Tipo Forestal Ciprés de las Guaitecas, con 159.334 ha, pero que representa el $27,5 \%$ del total nacional del tipo (CONAF, 2012) (Figura No 1).

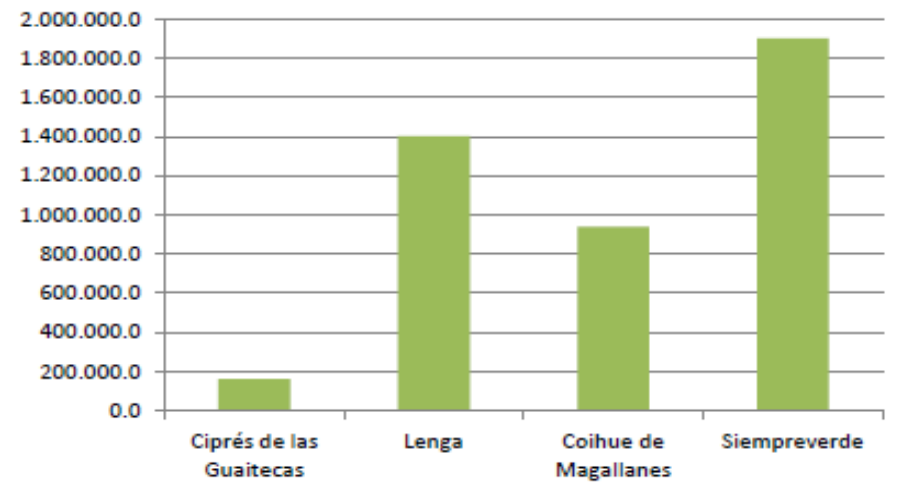

(Fuente: CONAF, 2012)

Figura $\mathrm{N}^{\circ} 1$

SUPERFICIE TIPOS FORESTALES DE LA REGIÓN DE AYSÉN

Pese a contar con un abundante recurso forestal, la industria del aserrío se encuentra muy por debajo del desarrollo industrial del resto de país, la representatividad de la industria del aserrío en comparación con el país solo alcanza al 3,8\% del total de aserraderos a nivel nacional (Cuadro $N^{\circ} 1$ ).

Respecto de la industria secundaria, que considera la elaboración o remanufactura, en la región tiene escaso desarrollo, representando esto una brecha a cubrir.

El secado de la madera constituye un punto clave en el proceso productivo posterior al aserrío, este es determinante en el tipo y calidad de los productos elaborados que se fabrican (Cubillos, 1987).

Durante la temporada 2017 la industria del aserrío en Aysén secó el 0,6\% (53 m³/año) de su producción en cámaras de secado (INFOR, 2018). 


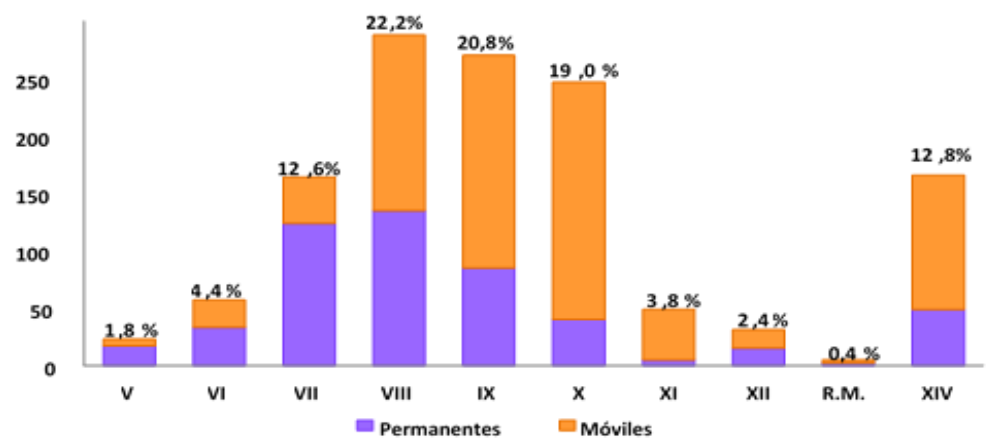

(Fuente: INFOR, 2018)

Figura $\mathrm{N}^{\circ} 2$

NÚMERO DE ASERRADEROS MÓVILES Y PERMANANTES POR REGIÓN

La industria regional de aserrío concentra su actividad principalmente en las Provincias de Aysén y Coyhaique (Cuadro $\mathrm{N}^{\circ} 1$ ), que representan el $40 \%$ y el $32 \%$, respectivamente, de la producción de madera aserrada durante el año 2017 (INFOR, 2018).

La producción de los aserraderos locales se comercializa principalmente como madera bruta y semiverde sin procesos de secado ni impregnación asociados, en venta directa, a pedidos, venta a intermediarios y venta a constructoras. De un total de 49 unidades productivas (aserraderos) en la Región de Aysén, solo el 6\% tiene asociado la elaboración de productos (forros, pisos, centros puertas y ventanas, traslapos) con una segunda línea de producción en sus instalaciones (INFOR, 2018).

\section{Cuadro $\mathrm{N}^{\circ} 1$}

REGISTRO PRODUCCIÓN DE MADERA REGIÓN DE AYSÉN (INFOR, 2018)

\begin{tabular}{|c|c|c|c|c|c|}
\hline \multirow{2}{*}{$\begin{array}{c}\text { Tipo de } \\
\text { Aserraderos y } \\
\text { Rango de } \\
\text { Producción }\end{array}$} & \multirow{2}{*}{ Total } & \multicolumn{4}{|c|}{ Producción por Provincia } \\
\hline & & Aysén & Capitán Prat & Coyhaique & $\begin{array}{l}\text { General } \\
\text { Carrera }\end{array}$ \\
\hline \multicolumn{6}{|c|}{$\left(\mathrm{m}^{3}\right)$} \\
\hline Total & 8.853 & 2.719 & 245 & 5.500 & 389 \\
\hline Permanente & 702 & 519 & 35 & 148 & - \\
\hline$<=5.000$ & 702 & 519 & 35 & 148 & - \\
\hline Móvil Portátil & 8.150 & 2.200 & 210 & 5.351 & 389 \\
\hline $1.000-3.000$ & 2.873 & - & - & 2.873 & - \\
\hline$<1.000$ & 5.277 & 2.200 & 210 & 2.478 & 389 \\
\hline
\end{tabular}

(Fuente: INFOR, 2018)

El análisis regional del rubro industrial maderero es decidor; requiere aumentar la capacidad de secado de madera, incorporar tecnologías más eficientes a los sistemas productivos, formación técnica, mayor capacitación y asistencia técnica, y potenciar los encadenamientos asociativos y la agregación de valor. 


\section{OBJETIVO}

Incorporar y validar tecnología de secado artificial para pequeñas producciones de madera, a través de un sistema de secado por deshumidificación.

\section{MATERIALES Y METODOS}

Las pruebas de secado de madera se realizaron con un sistema de deshumidificación adquirido por el Instituto Forestal a través de su Programa Prototipo de Transferencia Forestal (PTTF) financiado por el Gobierno Regional de Aysén.

El equipo corresponde a un deshumidificador marca WoodMizer, modelo DH4000 (Figura $\mathrm{N}^{\circ} 3$ ), el cual está diseñado para instalarlo en espacios herméticos y cerrados. Su capacidad máxima es de $9,4 \mathrm{~m}^{3}$ (395 pulgadas madereras).

El tiempo de secado según especificaciones técnicas, para piezas de 1 pulgada de espesor (1" x 10" × 3,2 m de largo) de pino (Pinus spp.) es de 12 días aproximadamente para $\mathrm{CH}$ inicial de $89 \%$ y final de $12 \%$.

Mientras que para piezas de iguales dimensiones para roble (Nothofagus obliqua), con $\mathrm{CH}$ inicial de $65 \%$ y final de $12 \%$, el tiempo de secado es de 35 días aproximadamente, esta especie posee características organolépticas ${ }^{15}$ similares a lenga y pinos de producción local (WoodMizer, 1982). a $220 \mathrm{~V} / 50 \mathrm{~Hz}$.

Este equipo de deshumidificación, tiene requerimientos eléctricos que van de $220 \mathrm{~V} / 60 \mathrm{~Hz}$
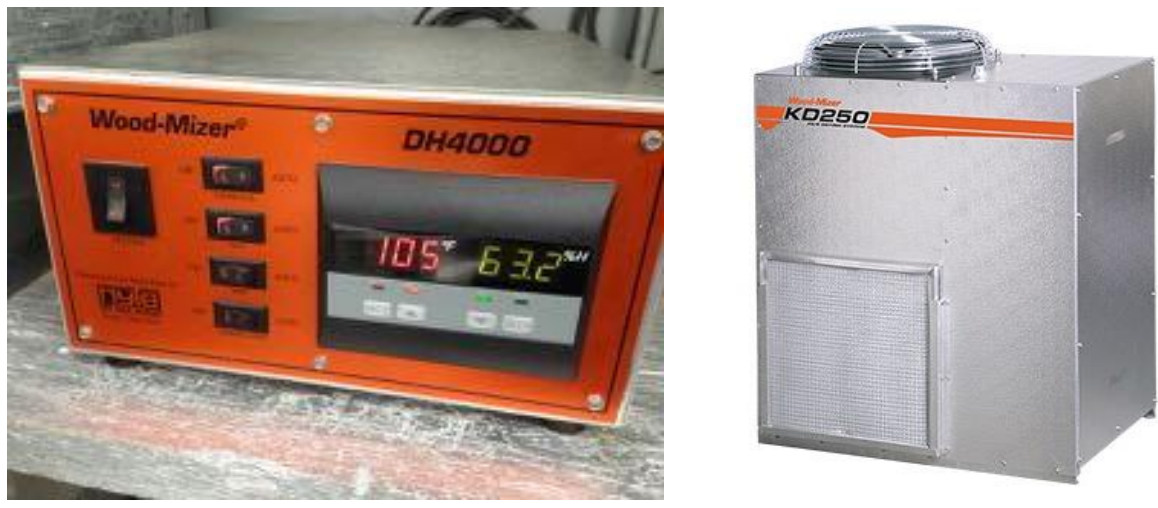

Figura $\mathrm{N}^{\circ} 3$ EQUIPO DESHUMIDIFICADOR MODELO DH4000 WOODMIZER

Para el funcionamiento de este equipo se requirió la habilitación de una estructura hermética que permitiera un secado óptimo (Figura $\mathrm{N}^{\circ} 4$ ). Para estos efectos, se estableció un convenio de cooperación con la empresa Maderas JHV Ltda., de propiedad del productor Javier Vargas Pérez, que proporcionó la madera y habilitó la infraestructura necesaria para el funcionamiento del equipo.

${ }^{15}$ Características generales que se relacionan con aspectos estéticos y de su estructura anatómica. 
El volumen requerido para el secado de madera fue de 350 a 400 pulgadas madereras por carga cada mes. Con este volumen, en la primera carga, fue posible calcular los costos en base a los tiempos y procedimientos de secado para comprobar la efectividad del proceso y los costos de funcionamiento, permitiendo así validar el secado de madera por deshumidificación como alternativa tecnológica para pequeñas producciones en la región.

La cámara de secado fue instalada en la propiedad del Sr. Javier Vargas, que se ubica en la comuna de Coyhaique, camino a Cerro Negro, km 1 (Figura $N^{\circ} 5$ ).

Habilitado el contendor, se procedió al llenado de la cámara en forma manual lo cual se podrá mejorar para posteriores cargas de madera con la incorporación de un sistema de carros y armado de paquetes en el exterior.
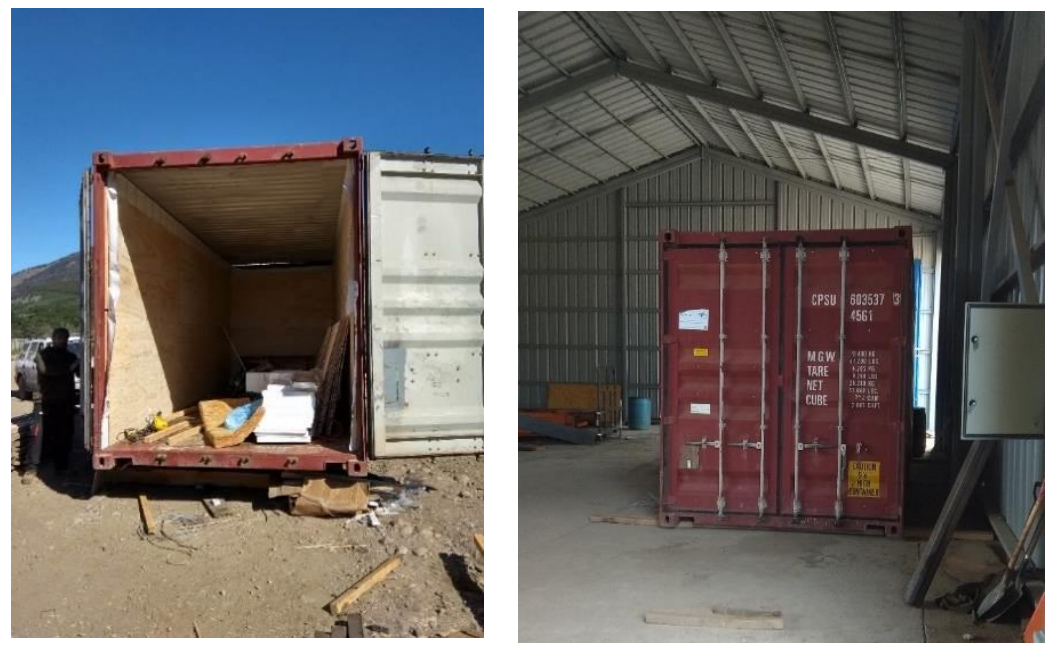

Figura $N^{\circ} 4$

ARMADO DE CONTENEDORES CON CÁMARA DE SECADO PARA INSTALACIÓN DE EQUIPO DESHUMIDIFICADOR
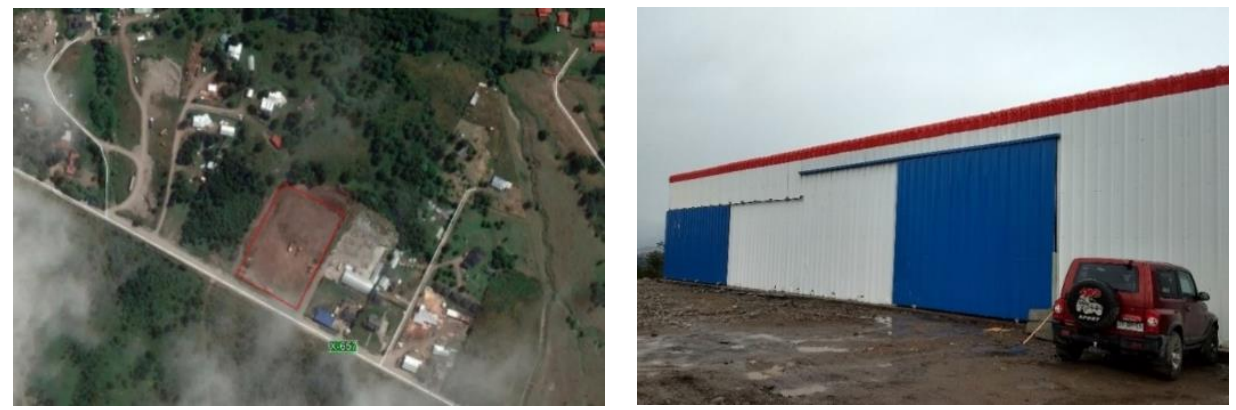

Figura $\mathrm{N}^{\circ} 5$

UBICACIÓN DE EMPRESA MADERAS JHV E INFRAESTRUCTURA DEL CONTENEDOR DE SECADO 


\section{Fases y Movimientos}

Para el carguío de madera al interior de la cámara de secado se contempló lo siguiente:

- Clasificación. Se aplica una clasificación visual además de una selección según escuadrías por anchos y largos, los espesores deben considerar espesores no mayores a $11 / 4 "$.

- $\quad$ Armado de castillos de madera. El correcto encastillado requiere listones homogéneos y secos, con distanciamientos no mayores a $50 \mathrm{~cm}$ uno del otro, que permitan el movimiento de aire.

- Medición de parámetros iniciales. La toma de muestras de contenido de humedad a la madera que ingresa es fundamental para que el ciclo de secado no sea fluctuante y de mayor tiempo.
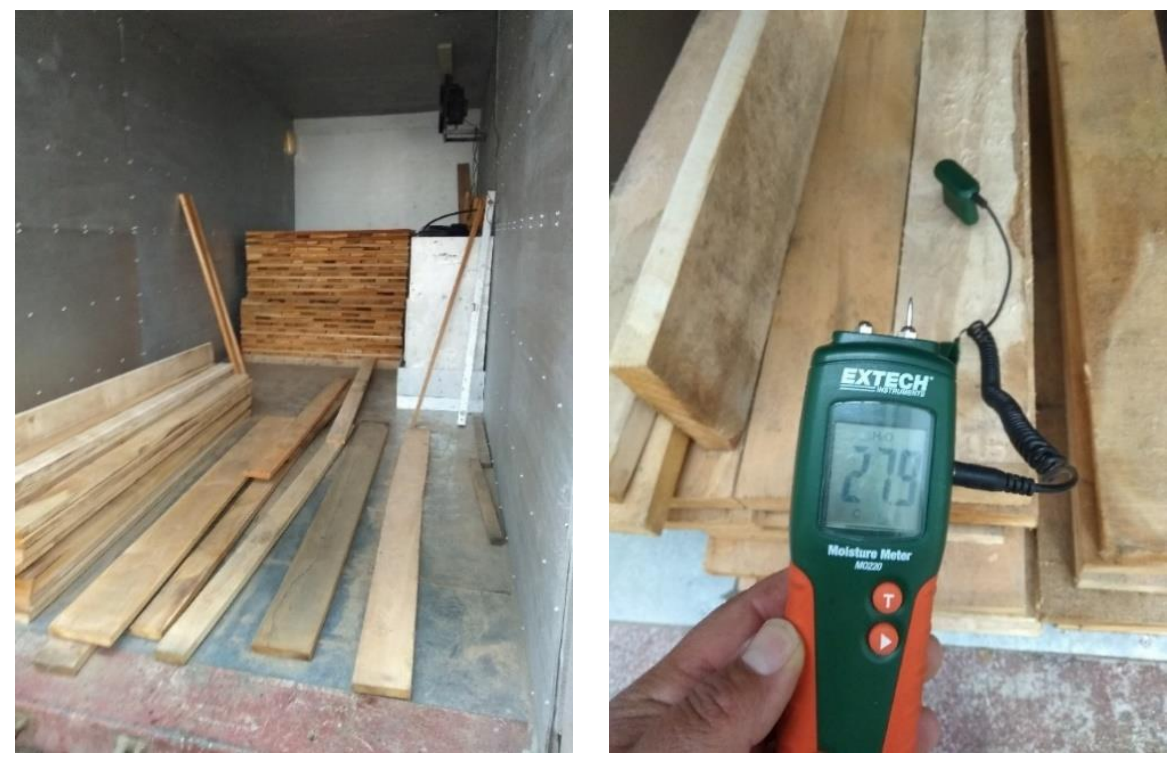

Figura $\mathrm{N}^{\circ} 6$

CLASIFICACION, ENCASTILLADO Y REGISTRO DE CONTENIDO DE HUMEDAD 

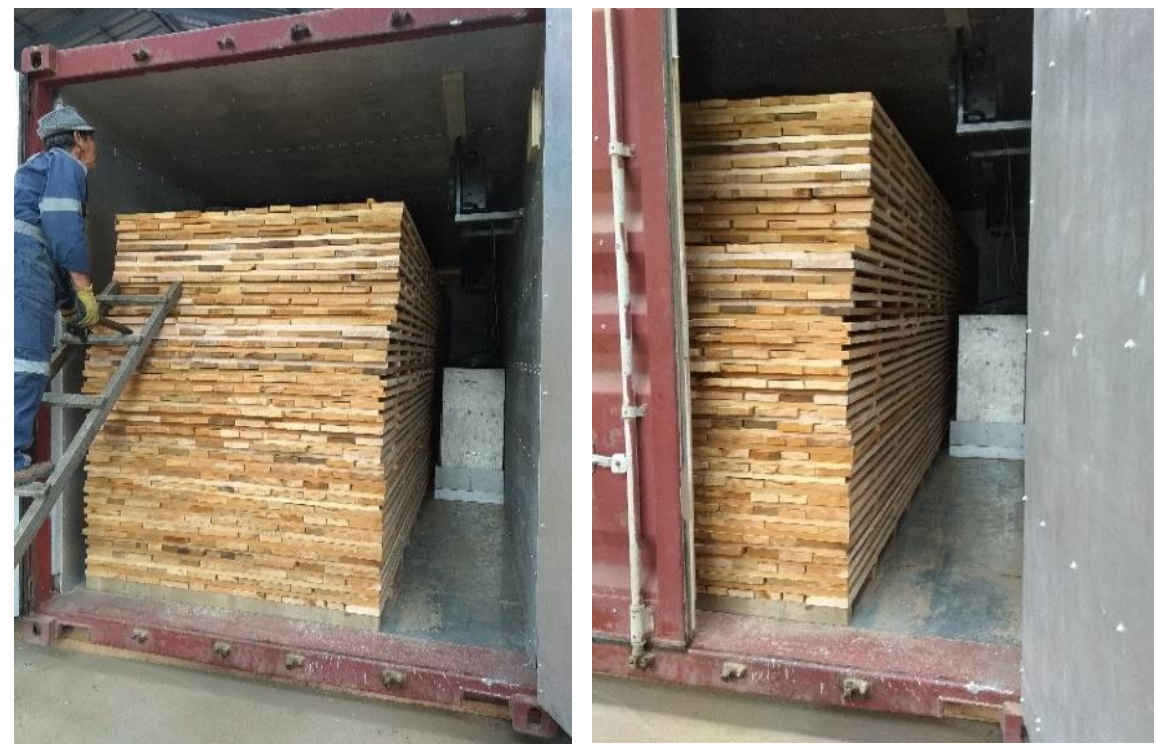

Figura $\mathrm{N}^{\circ} 7$

ENCASTILLADO

\section{Descripción de Parametros Inciales de Camara y Producto}

- $\quad$ Tipo de producto: Madera dimensionada

- Especie: Lenga

- Dimensión: Espesores calibrados a 1" nominal, 26 - 28 mm espesor, en anchos entre 4 5 pulgadas nominales $114-134 \mathrm{~mm}$.

- $\quad$ Contenido de humedad $(\mathrm{CH})$ promedio: $28 \%$

- Volumen: 380 pulgadas madereras

- $\quad$ Fecha ingreso de carga: 11 de abril de 2019

- $\quad$ Parámetros de medición del equipo: $52,7^{\circ} \mathrm{F}$ con $79,8 \%$ humedad.

- El carguío de la cámara se llevó a cabo con madera semiverde.

- $\quad$ Medición del CH inicial a 25 muestras

El objeto es producir madera seca de alto valor comercial que tienen gran demanda en el mercado local y nacional, siempre y cuando su humedad no supere el $12 \%$

Los tiempos de secado por deshumidificación no deberían superar los 30 días. El consumo del equipo es de 4,5- $5,0 \mathrm{~kW} / \mathrm{h}$ y el costo local de esta energía es de $\$ 250$ a $300 /$ por $\mathrm{KW} / \mathrm{h}$. Los costos de secado no deberían superar los $2.500 \$$ /" para pequeñas producciones de secado de especies nativas. 


\section{Control de Calidad y Monitoreo de la Humedad}

En la Figura $\mathrm{N}^{\circ} 8$ se detalla el proceso de monitoreo y contenido de humedad antes de ingreso a la cámara y el análisis de la información obtenida.

\begin{tabular}{|c|c|c|}
\hline \multicolumn{2}{|c|}{ INFOR SEDE PATAGONIA } & \\
\hline \multicolumn{2}{|c|}{ Nombre del Productor de Madera } & Javier Vargas \\
\hline \multicolumn{2}{|c|}{ Fecha de Muestreo } & 08-09-10 de abril \\
\hline \multicolumn{2}{|c|}{ Instrumento (Modelo, Marca) } & Xilohigrometro moisture meter MO220 \\
\hline \multicolumn{2}{|c|}{ Calibración (Temperatura, Densidad) } & \\
\hline \multicolumn{2}{|l|}{ Identificación Castillo } & lote 1 \\
\hline \multicolumn{2}{|c|}{$\begin{array}{l}\text { Descripción Castillo: (forma de } \\
\text { almacenamiento, especies principales, } \\
\text { esacudrias, etc) }\end{array}$} & $\begin{array}{l}\text { el almacenamiento se llevo a cabo en } \\
\text { castillo con separadores de } 1 / 2 \times 1 \\
\text { cada } 50 \mathrm{~cm} \text {. Los anchos estandar. }\end{array}$ \\
\hline \multicolumn{2}{|l|}{ Volumen del Lote } & 380 \\
\hline \multicolumn{2}{|l|}{ Escuadria (medida) } & 1" espesor nominal, 28-29 mm \\
\hline \multicolumn{2}{|l|}{ Encargado Monitoreo } & \\
\hline \multicolumn{2}{|l|}{ Especie } & Lenga \\
\hline \multicolumn{2}{|l|}{$N^{\circ}$ de Lote-Castillo } & 1 \\
\hline \multicolumn{2}{|l|}{ Fecha Elaboración } & ene-19 \\
\hline \multicolumn{2}{|l|}{ Procedencia } & Plan de Manejo, sector rio cajon. \\
\hline Muestra & $\begin{array}{l}\text { Ingrese Muestras } \\
\mathrm{CH}\end{array}$ & \\
\hline 1 & 17,0 & \\
\hline 2 & 27,0 & \\
\hline 3 & 28,0 & \\
\hline 4 & 32,0 & \\
\hline 5 & 33,0 & \\
\hline 6 & 26,0 & \\
\hline 7 & 27,0 & \\
\hline 8 & 22,0 & \\
\hline 9 & 27,0 & \\
\hline 10 & 26,0 & \\
\hline 11 & 27,0 & \\
\hline 12 & 28,0 & \\
\hline 13 & 36,0 & \\
\hline 14 & 34,0 & \\
\hline 15 & 32,0 & \\
\hline 16 & 33,0 & \\
\hline 17 & 26,0 & \\
\hline 18 & 23,0 & \\
\hline 19 & 29,0 & \\
\hline 20 & 20,0 & \\
\hline 21 & 32,0 & \\
\hline 22 & 34,0 & \\
\hline 23 & 22,0 & \\
\hline 24 & 29,0 & \\
\hline 25 & 30,0 & \\
\hline Humedad Prom. & 28,00 & \\
\hline Muestras Secas $(<25 \%)$ & 20,00 & \\
\hline Muestras Semihúmedas & 48,00 & \\
\hline Muestras Húm. $(>30 \%)$ & 32,00 & $N^{\circ}$ Muestra Optima \\
\hline Desviación St. & 4,73 & 11 \\
\hline Coef.Variación & 16,91 & \\
\hline Error de muestreo & 5,79 & $\%$ Error OK \\
\hline \multicolumn{3}{|c|}{ Nota 1: Considere que a mayor cantidad de muestras, mayor es la precisión de la medición } \\
\hline $\begin{array}{l}t-\text { student } \\
\text { n conf }\end{array}$ & $\begin{array}{r}n-1 \\
24\end{array}$ & $\begin{array}{l}\text { valor } \\
1,711\end{array}$ \\
\hline \multicolumn{3}{|c|}{ Lote rechazado, CH con $25 \%$ Inferior al $75 \%$ muestra } \\
\hline \multicolumn{3}{|c|}{ 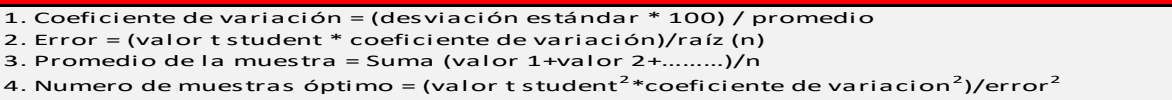 } \\
\hline
\end{tabular}

Figura $\mathrm{N}^{\circ} 8$

PLANILLA DE REGISTRO DE MEDICIONES DEL CONTENIDO HUMEDAD ANTES DEL INGRESO A LA CAMARA DE SECADO 
El equipo original (DH4000) fue reemplazado para terminar el proceso de secado, debido a problemas de funcionamiento con el lapso de apertura del compresor, por lo en que se cambió el equipo al octavo día de iniciado el ciclo de secado.

Por un periodo de 4 días se dejó ventilar la cámara para no provocar problemas de afloramiento de hongos en la madera. Luego de la instalación del nuevo equipo (KD250) el proceso se reanudó para terminar su ciclo de secado a los 10 días.

El equipo. adquirido por el productor, para terminar el proceso de secado fue un modelo KD250 (WoodMizer).

Este equipo es una versión moderna que genera un apagado automático al bajar al $12 \%$ de $\mathrm{CH}$, debido a que cuenta con sensores dispuestos al interior de la cámara que permiten un mejor registro, sin necesidad de abrir la cámara.

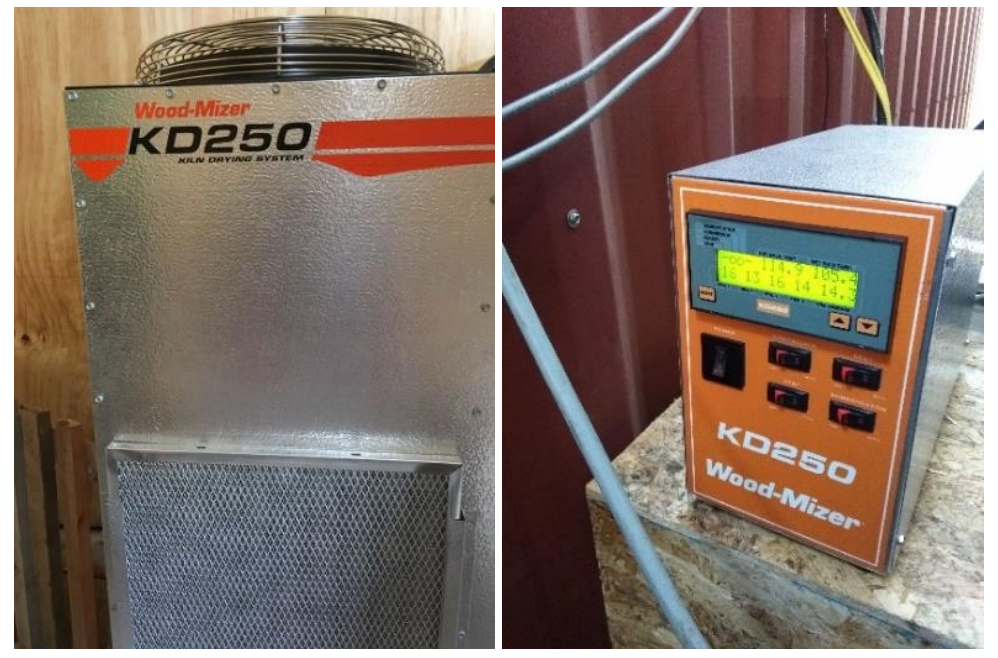

Figura $N^{\circ} 9$

EQUIPO KD250 WOODMIZER PARA TERMINAR EL PROCESO DE SECADO

\section{RESULTADOS}

El secado logrado fue $12 \% \mathrm{CH}$ en promedio al momento de terminar el ciclo, ocupando un tiempo de 18 días. Una vez extraída la madera se mantuvo bajo galpón para conservar estable el proceso de secado. Al final del proceso se obtuvo madera seca con $12 \% \mathrm{CH}$, este proceso permitió en 18 días bajar $16 \%$ el $\mathrm{CH}$ de la madera ingresada al $28 \% \mathrm{CH}$, calculándose una perdida máxima de $\mathrm{CH}$ por día de $0,8 \%$.

Considerando que en el proceso fue necesario cambiar el equipo por el KD250, el tiempo efectivo al no tener interrupciones debería tener periodos de secado no mayores a 12 días para, espesores de 25 a $29 \mathrm{~mm}$, lo que equivale a 1" nominal. La madera no presenta problemas de agrietado ni de torcedura (clasificación visual), esto se debe principalmente a que la curva de variación de calor es gradual y no alcanza a superar los $125^{\circ} \mathrm{F}\left(70^{\circ} \mathrm{C}\right)$.

A partir de la implementación del equipo se pudo tomar registro de la inversión inicial y la 
puesta en marcha de este, lo que se muestra en el Cuadro $\mathrm{N}^{\circ} 2$.

Cuadro $\mathrm{N}^{\circ} 2$

COSTOS DEL EQUIPAMIENTO NECESARIO PARA IMPLEMENTACION DEL SISTEMA DE SECADO

\begin{tabular}{|l|c|l|}
\hline Inversión & $\begin{array}{c}\text { Monto Invertido } \\
\text { (\$) }\end{array}$ & Condiciones de Operación \\
\hline $\begin{array}{l}\text { Equipo Deshumidificación KD250 } \\
\text { WoodMeizer. }\end{array}$ & 6.000 .000 & $\begin{array}{l}\text { Corriente monofásica con } \\
\text { dispositivo automático de 25 } \\
\text { amperes en el tablero }\end{array}$ \\
\hline $\begin{array}{l}\text { Instalaciones eléctricas y conexión del } \\
\text { equipo. }\end{array}$ & 1.000 .000 & \\
\hline $\begin{array}{l}\text { Implementación de aislación de contenedor } \\
\text { de dimensiones interiores de 2,3 } \times 2,3 \times 5,8 \\
\text { m. Esta implementación permite una } \\
\text { capacidad de secado de } 9 \mathrm{~m}^{3} .\end{array}$ & 4.500 .000 & $\begin{array}{l}\text { Forro aislación con plumavit } \\
\text { de } 50 \mathrm{~mm} \text {, papel filtro y } \\
\text { tablero de } 10 \mathrm{~mm} .\end{array}$ \\
\hline Total & $\mathbf{1 1 . 5 0 0 . 0 0 0}$ & \\
\hline
\end{tabular}

Para el cálculo de los costos de secado de madera en tanto (Cuadro $N^{\circ} 3$ ), se consideraron todas las actividades involucrados en el proceso y, a partir de su funcionamiento, se pudo estimar que el costo de secado por deshumidificación para una pulgada maderera fue $\$ 1.948$ (considerando una carga total de 380").

\section{Cuadro $N^{\circ} 3$}

\section{GASTOS IMPLICADOS EN EL SECADO DE MADERA POR DESHUMIDIFICACIÓN}

\begin{tabular}{|c|c|c|c|c|c|}
\hline Actividad & Unidad & Cantidad & $\begin{array}{c}\text { Costo } \\
\text { Unitario } \\
(\$)\end{array}$ & $\begin{array}{l}\text { Total } \\
(\$)\end{array}$ & Condiciones de Operación \\
\hline $\begin{array}{l}\text { Madera pre-seca } \\
\text { de lenga }\end{array}$ & $\begin{array}{l}\text { Pulgadas } \\
\text { madereras }\end{array}$ & 380 & 4.800 & 1.824 .000 & $\begin{array}{l}\text { Madera encastillada, valor de } \\
\text { amortización de } \mathrm{CH} \text { inicial de } \\
48 \% \text { y final (después de } 90 \\
\text { días) de } 28 \% \text { de } \mathrm{CH} \text {, en } \\
\text { medida de } 1 \text { " nominal. }\end{array}$ \\
\hline \multicolumn{4}{|l|}{ Subtotal } & 1.824 .000 & \\
\hline $\begin{array}{l}\text { Carguío } \\
\text { encastillado, } \\
\text { movimiento de } \\
\text { madera }\end{array}$ & Jornadas & 6 & 25.000 & 150.000 & $\begin{array}{l}2 \text { personas demoran } 3 \\
\text { jornadas en el llenado y } \\
\text { encastillado ocupando todo el } \\
\text { espacio. }\end{array}$ \\
\hline $\begin{array}{l}\text { Descarga y } \\
\text { extracción, } \\
\text { movimiento de } \\
\text { madera }\end{array}$ & Jornadas & 2 & 25.000 & 50.000 & $\begin{array}{l}2 \text { personas demoran } 1 \\
\text { jornada en descargar. }\end{array}$ \\
\hline $\begin{array}{l}\text { Consumo de } \\
\text { energía }\end{array}$ & $\mathrm{kW} / \mathrm{h}$ & 2.160 & 250 & 540.000 & $\begin{array}{l}\text { El consumo del equipo es } 4,5 \\
-5 \mathrm{~kW} / \mathrm{h} \text { (valor } \mathrm{kW} \\
\text { domiciliario } \$ 250) \text {. }\end{array}$ \\
\hline \multicolumn{4}{|l|}{ Subtotal } & 740.000 & \\
\hline \multicolumn{4}{|l|}{ TOTAL } & 2.564 .000 & \\
\hline
\end{tabular}




\section{CONCLUSIONES}

Generar información sobre el secado de madera a nivel regional ha sido una preocupación institucional, en 1972 existieron iniciativas de CORFO en Aysén con estudios sobre secado artificial de la especie lenga. Actualmente con la aparición de nuevas tecnologías, más eficientes y adaptadas a bajas producciones, se pueden encontrar diferentes opciones que permitan entregar soluciones a las necesidades de productores regionales.

El sistema de secado por deshumidificación implementado en la Región de Aysén, permitió validar una alternativa de secado que ofrece ciertas ventajas relacionadas a un problema básico que es la mano de obra calificada, ya que este equipo no requiere personal calificado para su operación.

Este proceso requiere disponer de madera con pre-secado y escuadrías no mayores a 2 pulgadas de espesor, lo que permite disminuir los costos y los daños por deformaciones.

El sistema de secado se ajusta a la realidad local, donde las unidades productivas trabajan con volúmenes bajos, permitiendo generar valor agregado y disponer de productos demandados en mueblería y en construcción.

Si se considera que la Región de Aysén cuenta con materia prima disponible y de alto valor, solo es necesario desarrollar y mejorar los estándares de calidad a partir del secado de madera, con el fin de alcanzar productos altamente competitivos en los mercados.

\section{RECONOCIMIENTOS}

Se agradece al Gobierno Regional de Aysén por el apoyo financiero que permitió desarrollar esta iniciativa que aporta a mejorar las capacidades de los productores forestales de la Región.

\section{REFERENCIAS}

CONAF, 2012. Catastro Vegetacional del Bosque Nativo. Superficies de Uso de Suelo Regional Actualización XI Región de Aysén. Coyhaique, Chile.

Cubillos, G., 1987. Costos del Secado por Deshumidificación. En: Ciencia e Investigación Forestal, Instituto Forestal, Chile. Vol. I, N², p. 121.

INFOR, 2018. Industria del Aserrío 2018. Instituto Forestal, Chile. Boletín Estadístico № 165. 158 pág.

WoodMizer, 1982. Introducción a los Sistemas de Secado en Horno. USA Indiana. 8p, 15p. 